\title{
The Central Vein: FLAIR Signal Abnormalities Associated with Developmental Venous Anomalies in Patients with Multiple Sclerosis
}

\author{
(DD.M. Rogers, DL.M. Shah, and (D) R.H. Wiggins III
}

\begin{abstract}
BACKGROUND AND PURPOSE: Demyelination is a recently recognized cause of FLAIR hyperintensities associated with developmental venous anomalies. Our purpose was to quantify the prevalence of white matter signal abnormalities associated with developmental venous anomalies in patients with multiple sclerosis compared with controls.
\end{abstract}

MATERIALS AND METHODS: A retrospective, blinded, multireader study compared the prevalence of FLAIR hyperintense signal abnormalities adjacent to developmental venous anomalies in patients with MS compared with controls (patients with developmental venous anomalies without MS). Study findings were positive if a central vein was demonstrated using FLAIR and contrast-enhanced fat-saturated T1 sequences. Imaging parameters also included developmental venous anomaly location, developmental venous anomaly drainage, white matter lesion size, and depth of white matter lesions. Clinical parameters included age, sex, and the presence of confounding variables (hypertension, diabetes, migraines, and/or vasculopathy).

RESULTS: FLAIR signal abnormality was present around 47.3\% (35/74) of developmental venous anomalies in patients with MS, and 13.5\% (10/74) of developmental venous anomalies in the control group $(P<.001)$. The multivariate logistic regression model controlling for covariates (including migraines, hypertension, diabetes mellitus, vasculopathy, age, sex, and drainage direction of developmental venous anomalies) showed that the odds of FLAIR hyperintensity around developmental venous anomalies was 6.7-fold higher in patients with MS (relative risk $\mathrm{MS}=6.68 ; 95 \% \mathrm{Cl}, 2.79-15.97 ; P<.001$ ).

CONCLUSIONS: The association of developmental venous anomalies and FLAIR hyperintensities was more common in patients with MS, which suggests that the underlying demyelinating pathologic process of MS may be the cause of this propensity in patients with MS. Impaired venous drainage in the territory of developmental venous anomalies may predispose to development of these lesions, and an associated central vein is helpful in understanding an atypical location of MS plaques.

ABBREVIATIONS: CCSVI = chronic cerebrospinal venous insufficiency; DVA $=$ developmental venous anomaly

D evelopmental venous anomalies (DVAs) are the most common congenital cerebral vascular malformation. ${ }^{1}$ They have a pathognomonic "umbrella" or "medusa" appearance on contrast-enhanced $\mathrm{T} 1$ or $\mathrm{T} 2{ }^{*}$ sequences due to numerous small medullary veins converging on a central collector vein, which may drain into a superficial cortical vein, dural venous sinus, or the deep venous system via a subependymal vein. ${ }^{2}$ Because DVAs are usually asymptomatic and represent the sole venous outflow to

Received May 31, 2018; accepted after revision August 9

From the Department of Radiology and Imaging Sciences, University of Utah Health Sciences Center, Salt Lake City, Utah.

Please address correspondence to Douglas M. Rogers, MD, 30 North 1900 East, \#1A071, Salt Lake City, UT 34132-2140; e-mail: Douglas.rogers@hsc.utah.edu; @rogersmdouglas

http://dx.doi.org/10.3174/ajnr.A5819 the brain parenchyma that they supply, they are known as "do not touch" lesions. ${ }^{3,4}$

Signal abnormalities on conventional FLAIR sequences are commonly observed in the drainage territory of DVAs. Prior studies have found the prevalence of white matter FLAIR hyperintensity near incidental DVAs to range from $11.6 \%$ to $30.7 \% .^{5-8}$ These FLAIR signal abnormalities tend to be larger and occur more frequently in older patients. ${ }^{5-8}$ Determining the etiology of the MR signal abnormalities around DVAs has been problematic because they are usually asymptomatic, and biopsy of such a lesion is unreasonably invasive. Edema or gliosis or both resulting from altered hemodynamics within the brain parenchyma drained by a DVA have been hypothesized as causes of the FLAIR signal abnormality around incidentally discovered DVAs, which is supported by findings on dynamic susceptibility contrast-enhanced perfusion-weighted imaging, in which CBV, CBF, TTP, and MTT are elevated. ${ }^{9-13}$ 
However, sequelae of demyelination have recently been recognized as another cause of signal abnormality surrounding DVAs in patients with multiple sclerosis. Two case series have described tumefactive demyelination occurring adjacent to DVAs in patients with MS. ${ }^{14,15}$ MS plaques have long been known to occur in a perivenular distribution (eg, Dawson fingers), and the development of 3T FLAIR ${ }^{\star}$ and 7T MR imaging has demonstrated that almost all MS plaques have a central vein. ${ }^{16-18}$ Gaitán et $\mathrm{al}^{17}$ showed on dynamic contrast-enhanced 7T MR imaging that small MS plaques enhance centrifugally from a central vein, and the "central vein sign" has been identified by the North American Imaging in Multiple Sclerosis Cooperative as an imaging feature with potential prospective diagnostic utility for the diagnosis of MS. ${ }^{18}$

DVAs are a common incidental finding on contrast-enhanced brain MR imaging. Two prior studies reported the prevalence of DVAs in patients with MS as $12.1 \%$ and $12.6 \%$, respectively. ${ }^{19,20}$ Our hypothesis is that demyelination is a pathologic cause of DVA-associated FLAIR hyperintensities in patients with MS. The purpose of this retrospective study was to compare the prevalence of DVA-associated FLAIR hyperintensities with a central vein in patients with MS and patients without MS who had incidentally detected DVAs. A greater prevalence of FLAIR signal abnormalities adjacent to DVAs in patients with MS compared with controls and/or the presence of other distinguishing features (such as lesion enhancement in patients with MS) would support the underlying demyelinating pathologic process of MS as the cause of this propensity in the MS group.

\section{MATERIALS AND METHODS}

This was an institutional review board-exempted retrospective study comparing the prevalence of white matter signal abnormalities on the FLAIR sequence adjacent to DVAs in patients with MS and patients without MS incidentally found to have DVAs.

\section{Subjects and Imaging}

Using a radiology report search engine (Montage; Nuance Communications, Burlington, Massachusetts), we searched brain MR imaging studies obtained at an academic university hospital using the terms "developmental venous anomaly (or DVA)" AND "multiple sclerosis (or MS)" for the study group, and "developmental venous anomaly (or DVA)" for the control group.

For inclusion criteria, FLAIR and T1 postcontrast images of adequate quality must have been available for interpretation, and a clearly delineated DVA must have been present. Additionally, for the study group, the patients must have had a clinical diagnosis of relapsing-remitting MS by a neurologist and met the revised 2017 McDonald criteria for MS diagnosis. ${ }^{21}$ Cases were excluded that had adjacent postoperative changes, vascular malformation, or hemorrhage (such as a cavernous malformation), as defined by susceptibility blooming artifacts adjacent to the DVA on gradient recalled-echo or susceptibility-weighted imaging.

For the study group, our search resulted in 81 DVAs found in 78 patients with MS with brain MR imaging studies performed between 2013 and 2017. One case was excluded due to an adjacent cavernous malformation, and 6 were excluded due to association with adjacent confluent white matter lesions (this imaging crite-
Table 1: Study and control population characteristics

\begin{tabular}{lccc}
\hline \multicolumn{1}{c}{ Characteristic } & $\begin{array}{c}\text { Multiple } \\
\text { Sclerosis } \\
(\boldsymbol{n}=74)\end{array}$ & $\begin{array}{c}\text { Controls } \\
(\boldsymbol{n}=74)\end{array}$ & $\begin{array}{c}\boldsymbol{P} \\
\text { Value }\end{array}$ \\
\hline Mean age (SD) (yr) & $45.1(13.5)$ & $50.7(18.4)$ & .036 \\
Sex: female & $49(66.2 \%)$ & $37(50.0 \%)$ & .045 \\
MRI field strength 3T & $18(24.3 \%)$ & $11(14.9 \%)$ & \\
MRI field strength 1.5T & $56(75.7 \%)$ & $63(85.1 \%)$ & \\
DVA location & & & \\
$\quad$ Lobar & $49(66.2 \%)$ & $51(68.9 \%)$ & \\
Basal ganglia & $6(8.1 \%)$ & $5(6.7 \%)$ & \\
Cerebellum & $18(24.3 \%)$ & $15(20.3 \%)$ & \\
$\quad$ Brain stem & $1(1.4 \%)$ & $3(4.1 \%)$ & \\
DVA drainage & & & \\
$\quad$ Superficial & $50(67.5 \%)$ & $46(62.1 \%)$ & \\
Deep & $21(28.4 \%)$ & $25(33.8 \%)$ & \\
Both & $3(4.1 \%)$ & $3(4.1 \%)$ & \\
Hypertension & $13(17.6 \%)$ & $15(20.3 \%)$ & \\
Diabetes & $3(4.1 \%)$ & $6(8.1 \%)$ & \\
Migraines & $10(13.5 \%)$ & $10(13.5 \%)$ \\
Intracranial vasculitis & $1(1.4 \%)$ & $1(1.4 \%)$ & \\
\hline
\end{tabular}

rion is discussed later in the Materials and Methods section). These exclusions left 74 DVAs in the final MS study population.

For the control group, the 78 most recent brain MR imaging cases (from March to September 2017) with a DVA and without MS were analyzed, yielding 79 clearly delineated DVAs in 78 patients. Five cases were excluded due to the presence of an adjacent cavernous malformation for a final cohort of 74 DVAs. These scans were obtained for myriad reasons, most commonly to evaluate a neoplasm elsewhere in the brain or as a staging scan for possible malignancy.

Diagnostic MR imaging was performed on Prisma, Aera, Avanto, and Espree 1.5T scanners, or Prisma and Verio 3T scanners (Siemens, Erlangen, Germany). Most scans were obtained with a magnetic field strength of $1.5 \mathrm{~T}$ ( $80.4 \%)$, and the remainder were obtained at $3 \mathrm{~T}(19.6 \%)$. There was no significant difference in the number of studies performed at $1.5 \mathrm{~T}$ and $3 \mathrm{~T}$ between the study and control groups (Table 1). While there was some mild variability, a typical 2D-FLAIR sequence had a TR of $9000 \mathrm{~ms}$ and a TE of $110 \mathrm{~ms}$ with 5-mm slice thickness and no gap. FLAIR sequences were performed with chemical fat saturation. Typical contrast-enhanced 2D-T1 sequences had a TR of $600 \mathrm{~ms}$ and a TE of $10 \mathrm{~ms}$ with $5-\mathrm{mm}$ slice thickness and no gap.

\section{Image Analysis and Chart Review}

Two radiologists, a senior neuroradiologist with 25 years of experience and a senior resident with 4 years of experience, performed a blinded and randomized review of the images on a PACS. In cases of disagreement, a second blinded neuroradiologist with 15 years of experience served as a tie-breaker. Criteria for a positive study were adapted from the central vein sign as described in the 2016 consensus statement from the North American Imaging in Multiple Sclerosis Cooperative. ${ }^{18}$ In contrast to criteria used in the literature in which FLAIR hyperintensity in the vicinity of the DVA was used, we decided to use a stricter central vein criterion as subsequently described because it is more specific for demyelination. While Sati et $\mathrm{al}^{18}$ assessed the central vein and associated FLAIR hyperintense plaques on a single $3 \mathrm{~T} \mathrm{FLAIR}^{\star}$ sequence, we localized lesions with side-by-side comparison of conventional FLAIR and $\mathrm{T} 1$ postcontrast images. 


\begin{tabular}{lccc}
\multicolumn{1}{c}{ Variable } & $\begin{array}{c}\text { Multiple } \\
\text { Sclerosis }(\boldsymbol{n}=\mathbf{7 4})\end{array}$ & $\begin{array}{c}\text { Controls } \\
(\boldsymbol{n}=\mathbf{7 4})\end{array}$ & $\boldsymbol{P}$ Value \\
\hline Positive FLAIR hyperintensity with a central vein & $35(47.3 \%)$ & $10(13.5 \%)$ & $<.001$ \\
FLAIR hyperintensity depth & & & \\
Juxtacortical & $13(37.1 \%)$ & $5(50.0 \%)$ & \\
Subcortical & $12(37.5 \%)$ & $3(30.0 \%)$ & \\
Periventricular & $10(28.6 \%)$ & $2(20.0 \%)$ & \\
Mean FLAIR hyperintensity width (SD) (mm) & $8.5(7.3)$ & $8.0(4.5)$ \\
Enhancing lesions & $4(11.4 \%)$ & $0(0 \%)$ \\
Location of DVA with associated FLAIR & & \\
$\quad$ hyperintensity & $29(82.9 \%)$ & $10(100 \%)$ & \\
Lobar & $2(5.7 \%)$ & $0(0 \%)$ \\
Basal ganglia & $4(11.4 \%)$ & $0(0 \%)$ \\
Cerebellum & $0(0 \%)$ & $0(0 \%)$ \\
Brain stem & & \\
Drainage of DVA with associated & & \\
FLAIR hyperintensity & $23(65.7 \%)$ & $6(60 \%)$ & \\
Superficial & $12(34.3 \%)$ & $2(20 \%)$ & \\
Deep & $0(0 \%)$ & $2(20 \%)$ & \\
Both & & \\
\hline
\end{tabular}

\section{Data Analysis}

Comparisons between the groups for dichotomous outcomes were performed using the $\chi^{2}$ test if the minimum expected cell frequency assumption was met $(80 \%$ of the cells have expected frequencies of at least 5 and no cell has an expected frequency of $<1$ ). The $\mathrm{McNe}$ mar test was used for paired data. For comparison of paired groups on an interval-scaled variable, a $t$ test was performed. Poisson regression was used for a binary outcome, with a robust variance estimate. A multivariate logistic regression model was created with positive FLAIR signal around DVAs as an outcome while controlling for covariates (including migraines, hypertension, diabetes mellitus, vasculopathy, age, sex, and DVA drainage direction). Interrater

Criteria for positive FLAIR signal abnormality adjacent to a DVA with a central vein were the following:

1) The DVA vessel must pass through the FLAIR hyperintense lesion so that it is directly abutting it, approximately equidistant from the edges of the lesion and passing through it at no more than 2 locations.

2) The FLAIR hyperintense lesion is at least $3 \mathrm{~mm}$ in all planes.

3) Studies were excluded if the DVA-associated FLAIR hyperintense lesion merged with another parenchymal lesion (eg, a superficial DVA extending toward multiple large periventricular plaques). While these studies might represent DVA-associated lesions, they were not included to avoid the possibility of chance associations with adjacent lesions in patients with MS who may have an overall higher burden of white matter disease.

4) Care was taken not to count flow-related FLAIR signal within the vessel itself as a surrounding parenchymal abnormality.

Imaging parameters included DVA location (lobar, basal ganglia, cerebellum, or brain stem), DVA drainage direction (superficial, deep, or both), the presence of an associated white matter lesion meeting the aforementioned central vein criteria, width of the white matter lesion measured in millimeters, and depth of the white matter lesion (juxtacortical, subcortical, or periventricular). The DVA drainage direction was defined as the confluent draining vessel extending either toward the surface of the brain (superficial), toward the ventricles (deep), or both. "Juxtacortical" was defined as a lesion that directly abuts the cortex, while a "periventricular" lesion directly abuts the lateral, third or fourth ventricles.

Clinical electronic medical record chart review was performed to confirm that study group patients had been diagnosed with MS by a neurologist and met the revised $2017 \mathrm{McD}$ onald criteria for the diagnosis of MS. Clinical parameters documented on the electronic medical record review included patient age and sex, duration since the time of diagnosis of MS, and potential confounding variables for white matter FLAIR hyperintensities such as hypertension, diabetes, migraines, and/or intracranial vasculitis. reliability was measured using the $\kappa$ coefficient, which is the proportion of agreement beyond expected chance agreement.

\section{RESULTS}

Study and control population characteristics are compared in Table 1 . Of note, the MS study group was slightly younger than the control population $(P=.036)$, with a female preponderance $(P=$ .045). There were no significant differences in MR imaging field strength, DVA location, or DVA drainage in the study and control populations. Clinical parameters, including the presence of hypertension, diabetes, migraines, and/or intracranial vasculitis, were also not significantly different in the study and control populations.

FLAIR signal abnormality meeting the central vein criteria was present adjacent to $47.3 \%$ (35/74) of DVAs in patients with MS and adjacent to $13.5 \%(10 / 74)$ of DVAs in the control group $(P<$ .001) (Table 2). A multivariate logistic regression model controlling for covariates (including migraines, hypertension, diabetes mellitus, vasculopathy, age, sex, and drainage direction of DVAs) showed that the odds of FLAIR hyperintensity around DVAs was 6.7-fold higher in patients with MS (relative risk MS = 6.68; 95\% CI, 2.79-15.97; $P<.001)$. In patients with MS, 11.4\% (4/35) of the lesions demonstrated enhancement compared with none in the control group. There was no significant difference in FLAIR signal surrounding DVAs according to sex in the patients with MS $(P=.504)$ or in the control subjects $(P=.197)$. There was a 1.4-fold increase in FLAIR signal surrounding DVAs in controls for every 10 -year increase in age (relative risk MS $=1.40 ; 95 \% \mathrm{CI}$, $1.01-1.94 ; P=.047)$. No such age-related association was present in the MS cohort. The mean number of years between the diagnosis of patients with MS and current imaging was $10.3 \pm 8.6$ years (range, 1-37 years). All except 4 patients with MS were on disease-modulating immunotherapy. Two of these patients had DVA-associated FLAIR hyperintensities, neither of which demonstrated enhancement. Examples of FLAIR hyperintense lesions adjacent to DVAs in patients with MS are shown in Fig 1, and 


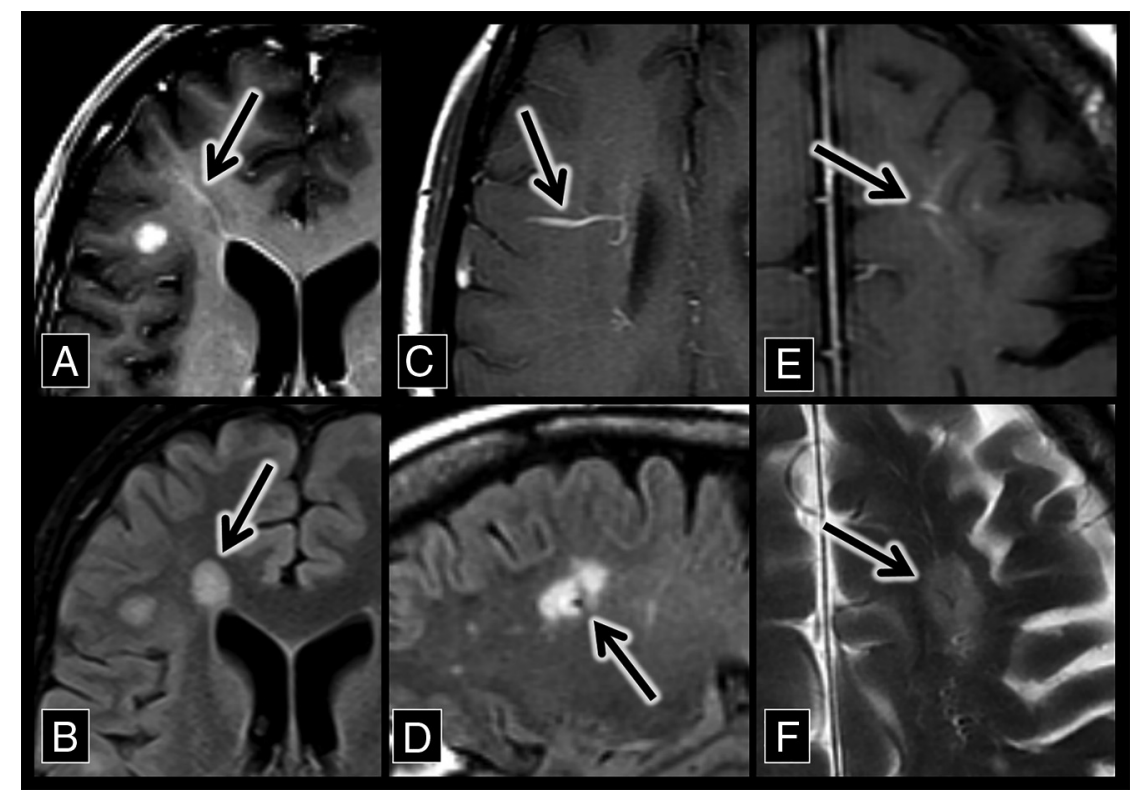

FIG 1. Developmental venous anomaly-associated lesions in patients with MS. A, An axial contrast-enhanced T1 sequence shows a right frontal lobe DVA (arrow) with surrounding T1 hypointensity. $B$, An axial FLAIR sequence shows hyperintensity (arrow) that corresponds to the DVA and associated TT hypointensity in $A$. C, An axial contrast-enhanced $T 1$ sequence shows a right frontal lobe DVA (arrow). D, A sagittal FLAIR sequence shows a flow void with adjacent hyperintensity (the central vein sign, arrow), which corresponds to the DVA in C.E, An axial contrast-enhanced T1 sequence shows a left frontal lobe DVA (arrow). F, An axial T2 sequence shows a flow void with adjacent hyperintensity (the central vein sign, arrow), which corresponds to the DVA in E.

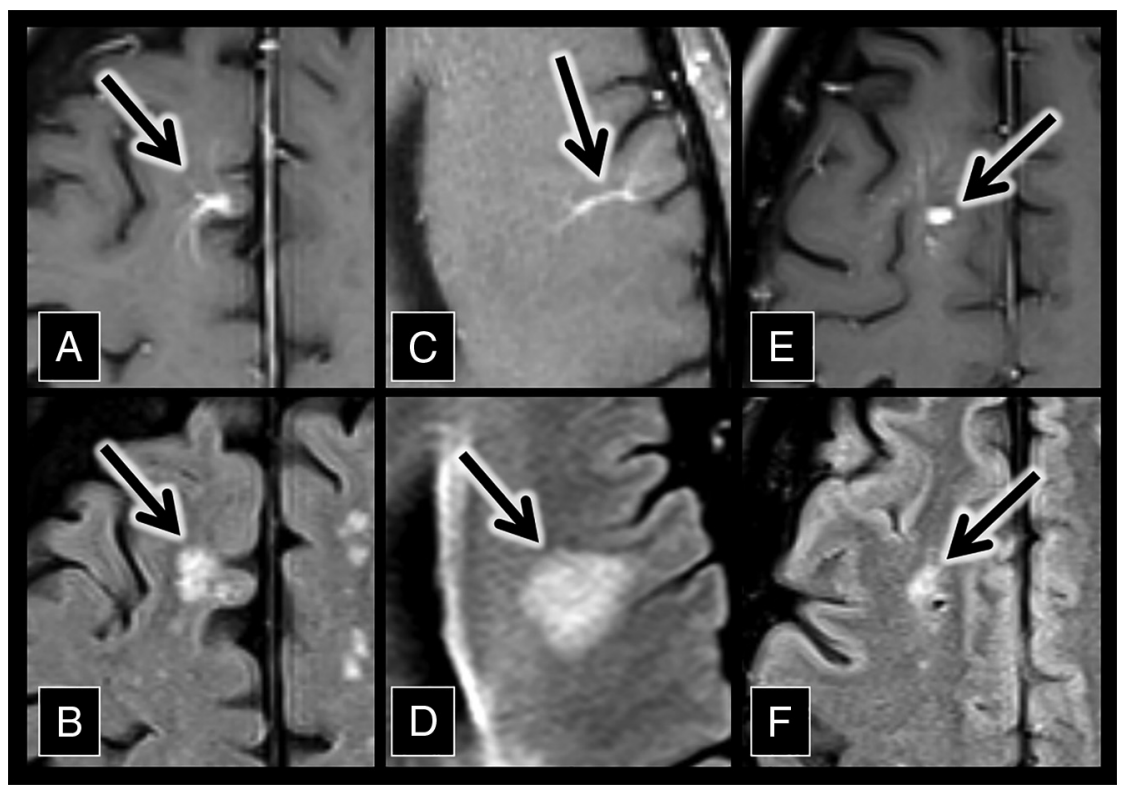

FIG 2. Developmental venous anomaly-associated lesions in the control group. A, An axial contrast-enhanced Tl sequence shows a right frontal lobe DVA (arrow). B, An axial FLAIR sequence shows hyperintensity (arrow) adjacent to the DVA in A. C, An axial contrast-enhanced Tl sequence shows a left frontal lobe DVA (arrow). D, An axial FLAIR sequence shows hyperintensity (arrow) adjacent to the DVA in C. D, An axial contrast-enhanced T7 sequence shows a right frontal lobe DVA (arrow). F, An axial FLAIR sequence shows hyperintensity (arrow) adjacent to the DVA in $E$.

FLAIR hyperintense lesions in the control group are shown in Fig 2.

For the cases of MS, the interrater reliability for the detection of FLAIR signal surrounding a DVA was 0.865 (95\% CI, $0.748-$ 0.979). For the control cases, the interrater reliability for detection of FLAIR signal surrounding a DVA was 0.874 (95\% CI, 0.702-1). This corresponds to excellent agreement. ${ }^{22}$

\section{DISCUSSION}

In our study, FLAIR signal abnormalities adjacent to DVAs were found to be significantly more prevalent in patients with MS compared with the control group of patients without MS (47.3\%$13.5 \%, P<.001)$. Multivariate logistic regression accounting for multiple possible confounding variables found that white matter lesions are 6.7 times more likely around DVAs in patients with MS compared with controls (95\% CI, 2.7915.97; $P<.001)$. The underlying pathologic process in both the control group and in some of the patients with MS is likely perivenular gliosis secondary to vascular congestion, venous hypertension, and chronic hypoxia in the territory of the DVA, as described in the neuropathology literature and previously depicted with MR perfusion. ${ }^{9-13,23-25}$ However, the significantly higher proportion of DVA-associated FLAIR abnormalities in patients with MS combined with the presence of lesion enhancement in some cases suggests an additional underlying pathologic cause (eg, sequelae of demyelination caused by multiple sclerosis) of these signal abnormalities in patients with MS. To be clear, these results do not imply that demyelination is the underlying cause of the signal abnormalities in the control group or healthy patients with DVAs. The underlying cause of the leukoaraiosis on FLAIR sequences in each case is indistinguishable when they are chronic lesions (Figs 1 and 2). However, advanced MR imaging techniques such as perfusion MR imaging have the potential of distinguishing DVA-associated FLAIR hyperintensity due to demyelination prospectively, and are an exciting avenue for further research.

The association of DVAs with demyelination in patients with MS has clinical utility, particularly in cases in which there is imaging overlap between active demyelination and possible central nervous system neoplasms (Fig 3). ${ }^{14}$ A noninvasive diagnostic strategy in these cases is short-term follow-up imaging because the enhancement of an actively demyelinating lesion will fade with time, persisting chronically as a DVA-associated FLAIR hyperintensity. The association of DVAs and FLAIR hyperintensities in 


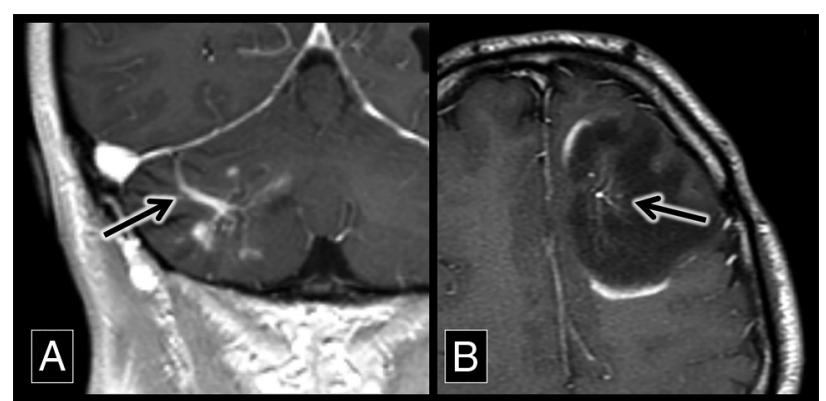

FIG 3. Demyelinating lesions around developmental venous anomalies with enhancement. $A$, A coronal contrast-enhanced $T 1$ sequence shows a superficially draining right cerebellar DVA (arrow) associated with enhancing parenchymal lesions. This was biopsy-proved demyelination. B, An axial contrast-enhanced $T 1$ sequence shows a left frontal lobe DVA (arrow) with surrounding $T 7$ hypointensity and discontinuous peripheral enhancement, typical of demyelination in a patient with MS.

patients with MS can also be useful to explain why demyelinating plaques may appear in atypical locations such as the basal ganglia. MS plaques classically appear along normally distributed veins (such as at the callososeptal interface) but also can be seen in areas of the brain where there is variant venous anatomy.

Previously reported theories may explain why FLAIR hyperintensities adjacent to DVAs are more common in patients with MS. The first is that patients with MS are predisposed to develop demyelination around DVAs due to their local inefficient venous drainage. ${ }^{9-13}$ MS plaques form when lymphocytes and monocytes cross the blood-brain barrier across venous channels, leading to perivenular cuffing. ${ }^{26,27}$ It is here that they react to autoantigens such as myelin basic protein, inciting an inflammatory response leading to perivenular demyelination. ${ }^{28,29}$ Jung et $\mathrm{al}^{9}$ previously showed on MR imaging perfusion that MTT and TTP are prolonged around most DVAs, suggestive of venous congestion in the parenchyma supplied by these DVAs. We hypothesize that the local venous congestion in the territory of a DVA predisposes to blood-brain barrier breakdown and lymphocytic infiltration, leading to demyelination in these regions. Previously published pathology-proved cases also support this hypothesis. ${ }^{14,30}$ It is unknown whether DVAs carry a greater risk for developing surrounding demyelination compared with normally distributed veins (such as callososeptal medullary veins). However, our finding that nearly half of DVAs in patients with MS have associated FLAIR hyperintensity, combined with the previously published findings supporting venous congestion in these regions, raises this possibility. Further investigation of DVA-associated signal abnormalities in patients with MS with MR imaging perfusion may help prove this theory.

It is critical that we address the controversial entity chronic cerebrospinal venous insufficiency (CCSVI), given the volume of medical literature and social media attention devoted to it. The CCSVI theory suggests that patients with MS are more likely to have stenosis of cerebrospinal venous outflow tracts, leading to intracranial venous reflux. ${ }^{31,32}$ In theory, a higher prevalence of global cerebral venous insufficiency in patients with MS superimposed on the impaired drainage of DVAs could lead to a higher rate of DVA-associated FLAIR hyperintensities in patients with MS compared with controls. However, CCSVI has largely been debunked as an underlying cause of MS lesions because there has been only a weak association between the CCSVI sonographic criteria proposed by Zamboni et $\mathrm{al}^{32}$ and MS, and multiple studies have found no statistically significant difference in venous drainage between patients with MS and controls. ${ }^{33-38}$ While global venous insufficiency in patients with MS seems unlikely on the basis of the current literature, this does not mean that local impairment of venous drainage in the territory of a DVA cannot be an exacerbating factor in MS.

There was an approximately 2:1 ratio of females to males in the MS group, while it was a 1:1 ratio in the control group. This ratio supports the previously reported epidemiology of MS. ${ }^{39}$ This difference was accounted for in the statistical analysis with a multivariate logistic regression model. We do not hypothesize that sex is an independent risk factor for the development of white matter lesions adjacent to DVAs, and sex predilection for these lesions has not been reported in the literature. In our study, of the 10 patients positive for DVA-associated FLAIR hyperintensity in the control group, 6 were male (60\%). We found no statistical correlation of sex and FLAIR hyperintensity surrounding DVAs in patients with MS or controls.

In our study, the MS group was slightly younger than the control group. This is likely because patients with MS present for imaging due to symptoms at a younger age than the background population. This discrepancy was accounted for in the statistical analysis with a multivariate logistic regression model. Additionally, we are reporting a higher prevalence of DVA-associated signal abnormalities in the younger MS group; it has been reported in the literature that these signal abnormalities are more common in older patients. ${ }^{5}$ Similarly, univariate regression analysis showed that older control patients were more likely to have FLAIR hyperintensities adjacent to DVAs $(P=.047)$. No association between patient age and the prevalence or size of DVAassociated signal abnormalities was seen in the MS group.

We acknowledge the limitations of this retrospective study. There may be a selection bias because a radiology report search tool was used for initial inclusion in the study. Cases in which smaller DVAs may not have been perceived by the prospectively interpreting radiologist or cases in which a DVA was identified but not included in a radiology report were not included in our study. We used a $\mathrm{T} 1$ postcontrast sequence to detect DVAs, which is potentially less sensitive compared with $\mathrm{T} 2{ }^{\star}$ sequences (such as SWI or 3T FLAIR ${ }^{\star}$ ). Additionally, our 5-mm slice thickness may also be less sensitive compared with thinner slice techniques. However, being present in both cohorts of patients somewhat mitigates these biases. One might hypothesize that because patients with MS may have more white matter disease compared with controls, a chance association with DVAs is more likely. However, this was corrected for by adherence to our exclusion criteria, which specifically omitted cases in which there were confluent white matter lesions adjacent to the DVA.

\section{CONCLUSIONS}

Because statistically more FLAIR hyperintensities were found to surround DVAs in patients with MS compared with controls, our results suggest that the underlying demyelinating pathologic process of MS may be the cause of this propensity in patients with MS. 
We hypothesize that impaired venous drainage in the territory of DVAs predisposes to the development of these lesions in patients with MS, and further prospective evaluation of these lesions with MR perfusion is a logical next step. This imaging feature is helpful in distinguishing tumefactive demyelinating lesions and central nervous system neoplasms and in understanding atypical locations of MS plaques at sites of variant venous anatomy.

\section{REFERENCES}

1. Sarwar M, McCormick WF. Intracerebral venous angioma: case report and review. Arch Neurol 1978;35:323-25 CrossRef Medline

2. Truwit CL. Venous angioma of the brain: history, significance, and imaging findings. AJR Am J Roentgenol 1992;159:1299-307 CrossRef Medline

3. Garner TB, Del Curling O Jr, Kelly DL Jr, et al. The natural history of intracranial venous angiomas. J Neurosurg 1991;75:715-22 CrossRef Medline

4. Töpper R, Jürgens E, Reul J, et al. Clinical significance of intracranial developmental venous anomalies. J Neurol Neurosurg Psychiatry 1999;67:234-38 CrossRef Medline

5. Santucci GM, Leach JL, Ying J, et al. Brain parenchymal signal abnormalities associated with developmental venous anomalies: detailed MR imaging assessment. AJNR Am J Neuroradiol 2008;29: 1317-23 CrossRef Medline

6. San Millán Ruíz D, Delavelle J, Yilmaz H, et al. Parenchymal abnormalities associated with developmental venous anomalies. Neuroradiology 2007;49:987-95 CrossRef Medline

7. Umino $\mathrm{M}$, Maeda M, Matsushima N, et al. High-signal-intensity abnormalities evaluated by $3 \mathrm{D}$ fluid-attenuated inversion recovery imaging within the drainage territory of developmental venous anomalies identified by susceptibility-weighted imaging at $3 \mathrm{~T}$. Jpn J Radiol 2014;32:397-404 CrossRef Medline

8. Linscott LL, Leach JL, Zhang B, et al. Brain parenchymal signal abnormalities associated with developmental venous anomalies in children and young adults. AJNR Am J Neuroradiol 2014;35: 1600-07 CrossRef Medline

9. Jung HN, Kim ST, Cha J, et al. Diffusion and perfusion MRI findings of the signal-intensity abnormalities of brain associated with developmental venous anomaly. AJNR Am J Neuroradiol 2014;35:1539-42 CrossRef Medline

10. Iv M, Fischbein NJ, Zaharchuk G, et al. Association of developmental venous anomalies with perfusion abnormalities on arterial spin labeling and bolus perfusion-weighting imaging. J Neuroimaging 2015;25:243-50 CrossRef Medline

11. Camacho DL, Smith JK, Grimme JD, et al. Atypical MR imaging perfusion in developmental venous anomalies. AJNR Am J Neuroradiol 2004;25:1549-52 Medline

12. Sharma A, Zipfel GJ, Hildebolt C, et al. Hemodynamic effects of developmental venous anomalies with and without cavernous malformations. AJNR Am J Neuroradiol 2013;34:1746-51 CrossRef Medline

13. Hanson EH, Roach CJ, Ringdahl EN, et al. Developmental venous anomalies: appearance on whole-brain CT digital subtraction angiography and CT perfusion. Neuroradiology 2011;53:331-41 CrossRef Medline

14. Rogers DM, Peckham ME, Shah LM, et al. Association of developmental venous anomalies with demyelinating lesions in patients with multiple sclerosis. AJNR Am J Neuroradiol 2018;39:97-101 CrossRef Medline

15. Ma M, Chen JY, Plowey ED, et al. Tumefactive demyelination associated with developmental venous anomaly: report of two cases. Clin Imaging 2017;43:194-98 CrossRef Medline

16. Samaraweera AP, Clarke MA, Whitehead A, et al. The central vein sign in multiple sclerosis lesions is present irrespective of the T2* sequence at 3 T. J Neuroimaging 2017;27:114-21 CrossRef Medline
17. Gaitán MI, Sati P, Inati SJ, et al. Initial investigation of the bloodbrain barrier in MS lesions at 7 Tesla. Mult Scler 2013;19:1068-73 CrossRef Medline

18. Sati $\mathrm{P}, \mathrm{Oh} \mathrm{J}$, Constable $\mathrm{RT}$, et al. The central vein sign and its clinical evaluation for the diagnosis of multiple sclerosis: a consensus statement from the North American Imaging in Multiple Sclerosis Cooperative. Nat Rev Neurol 2016;12:714-22 CrossRef Medline

19. Zhao G, Li DK, Traboulsee T, et al. Developmental venous anomalies (venous angiomas) on MRI are more common in patients with multiple sclerosis. In: Proceedings of the International Society for Magnetic Resonance in Medicine Scientific Meeting and Exhibition and the Annual Meeting of the Society for MR Radiographers \& Technologists, Kyoto, Japan. May 15-21, 2004

20. Sasani MR, Dehghan AR, Ali Reza N. The relationship of multiple sclerosis and cerebral developmental venous anomaly with an advantageous role in the multiple sclerosis diagnosis. Iran J Neurol 2017;16:168-72 Medline

21. Thompson AJ, Banwell BL, Barkhof F, et al. Diagnosis of multiple sclerosis: 2017 revisions of the McDonald criteria. Lancet Neurol 2018;17:162-73 CrossRef Medline

22. Landis JR, Koch GG. The measurement of observer agreement for categorical data. Biometrics 1977;33:159-74 CrossRef Medline

23. Moody DM, Brown WR, Challa VR, et al. Periventricular venous collagenosis: association with leukoaraiosis. Radiology 1995;194: 469-76 CrossRef Medline

24. Keith J, Gao FQ, Noor R, et al. Collagenosis of the deep medullary veins: an underrecognized pathologic correlate of white matter hyperintensities and periventricular infarction? J Neuropathol Exp Neurol 2017;76:299-312 CrossRef Medline

25. Brown WR, Moody DM, Challa VR, et al. Venous collagenosis and arteriolar tortuosity in leukoaraiosis. J Neurol Sci 2002;203-204: 159-63 CrossRef Medline

26. Matthews PM, Roncaroli F, Waldman A, et al. A practical review of the neuropathology and neuroimaging of multiple sclerosis. Pract Neurol 2016;16:279-87 CrossRef Medline

27. Tanaka R, Iwasaki Y, Koprowski H. Ultrastructural studies of perivascular cuffing cells in multiple sclerosis brain. Am J Pathol 1975;81:467-78 Medline

28. Adams CW. The onset and progression of the lesion in multiple sclerosis. J Neurol Sci 1975;25:165-82 CrossRef Medline

29. Minagar A, Alexander JS. Blood-brain barrier disruption in multiple sclerosis. Mult Scler 2003;9:540-49 CrossRef Medline

30. Jung G, Schröder R, Lanfermann H, et al. Evidence of acute demyelination around a developmental venous anomaly: magnetic resonance imaging findings. Invest Radiol 1997;32:575-77 CrossRef Medline

31. Singh AV, Zamboni P. Anomalous venous blood flow and iron deposition in multiple sclerosis. J Cereb Blood Flow Metab 2009;29: 1867-78 CrossRef Medline

32. Zamboni P, Galeotti R, Menegatti E, et al. Chronic cerebrospinal venous insufficiency in patients with multiple sclerosis. J Neurol Neurosurg Psychiatry 2009;80:392-99 Medline

33. Zamboni P, Menegatti E, Galeotti R, et al. The value of cerebral Doppler venous haemodynamics in the assessment of multiple sclerosis. J Neurol Sci 2009;282:21-27 CrossRef Medline

34. Krogias C, Schröder A, Wiendl H, et al. "Chronic cerebrospinal venous insufficiency" and multiple sclerosis: critical analysis and first observation in an unselected cohort of MS patients [in German]. Nervenarzt 2010;81:740-46 CrossRef Medline

35. Doepp F, Paul F, Valdueza JM, et al. No cerebrocervical venous congestion in patients with multiple sclerosis. Ann Neurol 2010;68: 173-83 CrossRef Medline Medline

36. Comi G, Battaglia MA, Bertolotto A, et al; CoSMo Collaborative Study Group. Observational case-control study of the prevalence of chronic cerebrospinal venous insufficiency in multiple sclerosis: results from the CoSMo study. Mult Scler 2013;19:1508-17 CrossRef Medline 
37. Traboulsee AL, Knox KB, Machan L, et al. Prevalence of extracranial venous narrowing on catheter venography in people with multiple sclerosis, their siblings, and unrelated healthy controls: a blinded, case-control study. Lancet 2014;383:138-45 CrossRef Medline

38. Jedynak W, Cieszanowski A. Is there any relation between chronic cerebrospinal venous insufficiency and multiple sclerosis? A critical review. Pol J Radiol. 2014;79:131-36 CrossRef Medline

39. Alonso A, Hemán MA. Temporal trends in the incidence of multiple sclerosis: a systematic review. Neurology 2008;71:129-35 CrossRef Medline 